\title{
Patiromer in a Patient with Severe Hyperkalemia on Incremental Hemodialysis with 1 Session per Week: A Case Report and Literature Review
}

\author{
José C. De La Flor ${ }^{a}$ Javier Deirab Alexander Marschallc ${ }^{\mathrm{b}}$ Francisco Valgad $^{d}$ \\ Tania Linares $^{a}$ Tania Monzon $^{\text {e }}$ Cristina Albarracín ${ }^{a} \quad$ Elisa Ruiz ${ }^{a}$ \\ aDepartment of Nephrology, Central Defense Gomez Ulla Hospital, Madrid, Spain; \\ ${ }^{b}$ Department of Nephrology, San Pedro de Alcantara Hospital, Caceres, Spain; 'Department \\ of Cardiology, Central Defense Gomez Ulla Hospital, Madrid, Spain; dDepartment of \\ Nephrology, Doctor Negrín University Hospital, Las Palmas de Gran Canarias, Las Palmas, \\ Spain; eDepartment of Hemodialysis, Avericum S.L., Las Palmas de Gran Canarias, Las \\ Palmas, Spain
}

Keywords

Hyperkalemia · Incremental hemodialysis · Patiromer sorbitex calcium

\begin{abstract}
Hyperkalemia is common in patients with ESRD, undergoing hemodialysis (HD), and is associated with an increase in hospitalization and mortality. Residual kidney function in long-term dialysis patients is associated with lower morbidity and mortality in HD patients. Although the 2015 National Kidney Foundation-Kidney Disease Outcomes Quality Initiate (NKD-KDOQI) guidelines allow the reduction in the weekly HD dose for patients with a residual kidney urea clearance (Kur) $>3 \mathrm{~mL} / \mathrm{min} / 1.73 \mathrm{~m}^{2}$, very few centers adjust the dialysis dose based on these criteria. In our center, the pattern of incremental hemodialysis (iHD) with once-a-week schedule $(1 \mathrm{HD} / \mathrm{W})$ has been an option for a group of patients showing very good results. This pattern is maintained as long as residual diuresis is $>1,000 \mathrm{~mL} / 24 \mathrm{~h}$, Kur is $>4 \mathrm{~mL} / \mathrm{min}$, and there is no presence of edema or volume overload, as well as no analytical parameters persistently outside the advisable range (serum phosphorus $>6 \mathrm{mg} / \mathrm{dL}$ or potassium $\left[\mathrm{K}^{+}\right]>6.5 \mathrm{mmol} / \mathrm{L}$ ). Management of hyperkalemia in HD patients includes reduction of dietary intake, dosing of medications that contribute to hyperkalemia, and use of cation-exchange resins such as cal-
\end{abstract}

José C. De La Flor, Javier Deira, and Alexander Marschall take responsibility for all aspects of the reliability and freedom from bias of the data presented and their discussed interpretation. 
De La Flor et al.: Patiromer in a Patient with Severe Hyperkalemia on Incremental Hemodialysis

cium or sodium polystyrene sulfonate. Two newer potassium binders, patiromer sorbitex calcium and sodium zirconium cyclosilicate, have been safely used for potassium imbalance treatment in patients with ESRD in HD with a conventional regimen of thrice weekly, but has not yet been studied in $1 \mathrm{HD} / \mathrm{W}$ schedules. We present the case of a 76-year-old woman in iHD $(1 \mathrm{HD} / \mathrm{W})$ treated with patiromer for severe HK and describe her clinical characteristics and outcomes. In addition, we review the corresponding literature. Based on these data, it can be anticipated that the use of patiromer may overcome the risk of hyperkalemia in patients with incident ESRD treated with less-frequent HD regimens.

(C) 2021 The Author(s).

Published by S. Karger AG, Basel

\section{Introduction}

Hyperkalemia in hemodialysis (HD) patients is defined as a serum concentration of predialysis potassium $\left(\mathrm{K}^{+}\right)>5.5 \mathrm{mmol} / \mathrm{L}$, as determined in the extracellular compartment. Due to alterations in the cardiac conduction system which can lead to arrhythmias and sudden cardiac death, hyperkalemia is a potential life-threatening emergency in maintenance hemodialysis (MHD) patients, with every increase of $1 \mathrm{mmol} / \mathrm{L}$ above $5.1 \mathrm{mmol} / \mathrm{L}$ resulting in an increase of $40 \%$ in risk for sudden cardiac death [1]. Hyperkalemia in MHD patients is associated with an all-cause mortality of 3.1/1,000 patients per year [2-4]. Hyperkalemia is an extremely prevalent alteration in HD patients. However, few studies investigate this entity in conventional HD schemes, as well as in incremental hemodialysis (iHD). Data on its prevalence are inconsistent, reporting a prevalence between 5 and $20 \%$, depending on the size and characteristics of the study populations, as well as on the definition of predialysis hyperkalemia ( $\geq 5.1, \geq 5.5$, or $\geq 6 \mathrm{mmol} / \mathrm{L}$ ) and comorbidities, such as diabetes, heart failure, use of renin-angiotensin-aldosterone system inhibitors (RAASi).

In most developed countries, the vast majority of patients with ESRD receiving HD are treated with a conventional regimen of thrice weekly for 3-5 h per session. The residual kidney function (RKF) in long-term dialysis patients is associated with lower morbimortality in both peritoneal dialysis and HD patients [5, 6]. The 2015 National Kidney Foundation-Kidney Disease Outcomes Quality Initiate (NKF-KDOQI) guidelines allow the reduction in the weekly HD dose for patients with kidney urea clearance (Kur) $>3 \mathrm{~mL} / \mathrm{min} / 1.73 \mathrm{~m}^{2}$ [6]. Despite this, very few centers adjust the dialysis dose based on these criteria. Recent ongoing studies and clinical trials on the safety and efficacy of lessfrequent HD in patients with incident CKD have appeared in recent years, most of which consist of twice-weekly HD regimens at dialysis initiation [7-10], coinciding with the experience already published by Kalantar-Zadeh et al. $[9,10]$ in the USA and TeruelBriones et al. [11] and Merino et al. [12] in Spain. However, only one study, IHDIP trial led by Deira et al. [7], which is currently in the patient enrollment phase, proposes the once-a-week schedule.

The management of hyperkalemia in HD includes reduction of dietary intake, dosing of medications that contribute to hyperkalemia, and use of cation-exchange resins such as calcium or sodium polystyrene sulfonate. Due to gastrointestinal (GI) complications, such as constipation, diarrhea, and nausea, resins are mostly not well tolerated. Furthermore, its use is associated with potential life-threatening side effects, such as intestinal necrosis [13-15]. Recently, 2 new therapeutic options have been approved: patiromer sorbitex calcium and sodium zirconium cyclosilicate. The underlying pharmacologic mechanism of these drugs is the elimination of potassium in the GI tract, based on the exchange of $\mathrm{K}^{+}$for other cations

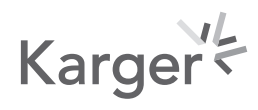


De La Flor et al.: Patiromer in a Patient with Severe Hyperkalemia on Incremental Hemodialysis

(calcium and sodium, respectively). Patiromer has shown to effectively reduce serum potassium levels [16], to maintain long-term normokalemia [17], and to prevent hyperkalemia in MHD patients with a conventional regimen of thrice weekly [18]. Based on these studies, the use of patiromer can be considered to avoid the risk of hyperkalemia, enhance the tolerance of RAASi, and maintain the HD prescription scheme in patients with iHD treated with less-frequent HD regimens. We report a case of a patient in iHD with once-a-week schedule treated with patiromer for severe hyperkalemia.

\section{Case Presentation}

We present the case of a 76-year-old woman with a history of arterial hypertension, hyperuricemia, and type 2 diabetes mellitus, diagnosed at the age of 60 years, with microvascular (CKD and retinopathy) and macrovascular (peripheral artery disease) affection. The patient has undergone follow-up in our Nephrology outpatient clinic for CKD stage G3b/A3, secondary to diabetic nephropathy, since 2010. The patient has had baseline serum creatinine values around $2.0 \mathrm{mg} / \mathrm{dL}$ and baseline urea of $90 \mathrm{mg} / \mathrm{dL}$. Due to progression of her disease to a terminal stage, the patient has undergone follow-up in our outpatient clinic for ESRD since 2019. In May 2020, the patient initiated HD via a native humerocephalic artery-venous fistula, presenting uremic symptoms, a serum creatinine of $7.8 \mathrm{mg} / \mathrm{dL}$ (estimated glomerular filtration rate, using the CKD-EPI formula, of $5.8 \mathrm{~mL} /$ $\mathrm{min} / 1.73 \mathrm{~m}^{2}$ ), a 24 -h creatinine clearance of $7.6 \mathrm{~mL} / \mathrm{min}, \mathrm{K}^{+}$of $5.5 \mathrm{mmol} / \mathrm{L}$, and a urine albumin/creatinine ratio of $733.99 \mathrm{mg} / \mathrm{g}$. Since the patient met the inclusion criteria: Kur $>4.5 \mathrm{~mL} / \mathrm{min} / 1.73 \mathrm{~m}^{2}$ in $24 \mathrm{~h}$ of RKF and urine volume $>500 \mathrm{~mL} / 24 \mathrm{~h}$, no active neoplasia, no heart failure in functional class NYHA IV, no unstable angina pectoris, no myocardial infarction in the previous 3 months, no treatment with immunosuppressors, nor any kind of active inflammatory disease, the patient was included in our iHD protocol with 1 session per week.

Two weeks after the initiation of the $\mathrm{HD}$, a progressive increase of the $\mathrm{SK}^{+}$levels, with a maximum level of $7.1 \mathrm{mmol} / \mathrm{L}$, was observed (Table 1). Venous blood gas analysis revealed a metabolic acidosis, despite treatment with bicarbonate, which justified the hyperkalemia. On the other hand, the patient did not present vascular complications, and the dialysis parameters were correct, with no variations in the previous 2 months (online hemofiltration with convective volumes of 20-27 L/session and KT of 53-57 L). Furthermore, the RKF controls with biochemical analysis of the 24-h urine maintained the Kur without changes, as well as the urine volume remained stable. We ruled out causes of pseudohyperkalemia (i.e., hemolysis caused by rotational blood pump or other components of the extracorporeal circuit, as well as rhabdomyolysis). The patient has been maintaining an adequate diet (low in $\mathrm{K}^{+}$) with a potassium uptake of approximately $30-40 \mathrm{mmol} / \mathrm{day}$. In addition to a potassium-restricted diet, an increase of the dose of PSC to a dose of $99.75 \mathrm{~g} / 100 \mathrm{~g} 24 \mathrm{~h}$ was necessary. Furthermore, we decreased the dose of RAASi by half and doubled the dose of the diuretics and calcium channel blockers. Despite these measures, the patient maintained high potassium levels, which is why the potassium concentration in the dialysis solution was reduced (from 2 to 1 $\mathrm{mmol} / \mathrm{L}$ ). However, the patient maintained potassium levels above $6 \mathrm{mmol} / \mathrm{L}$. Despite clinical signs of PSC intolerance, an attempt was made to increase the dose of PSC. However, the patient presented important GI symptoms, such as constipation and abdominal pain. Therefore, PSC was discontinued, and patiromer $8.4 \mathrm{~g}$ was initiated. Posterior analytic controls revealed predialysis potassium levels of $4.7 \mathrm{mmol} / \mathrm{L}$, and the patient did not complain of GI symptoms. We decided to increase the RAASi dose. One month later, the patient's biochemical analysis showed a potassium level of $4.9 \mathrm{mmol} / \mathrm{L}$, and in further controls, the

\section{Karger'}


Case Reports

in Nephrology and Dialysis
Case Rep Nephrol Dial 2021;11:158-166

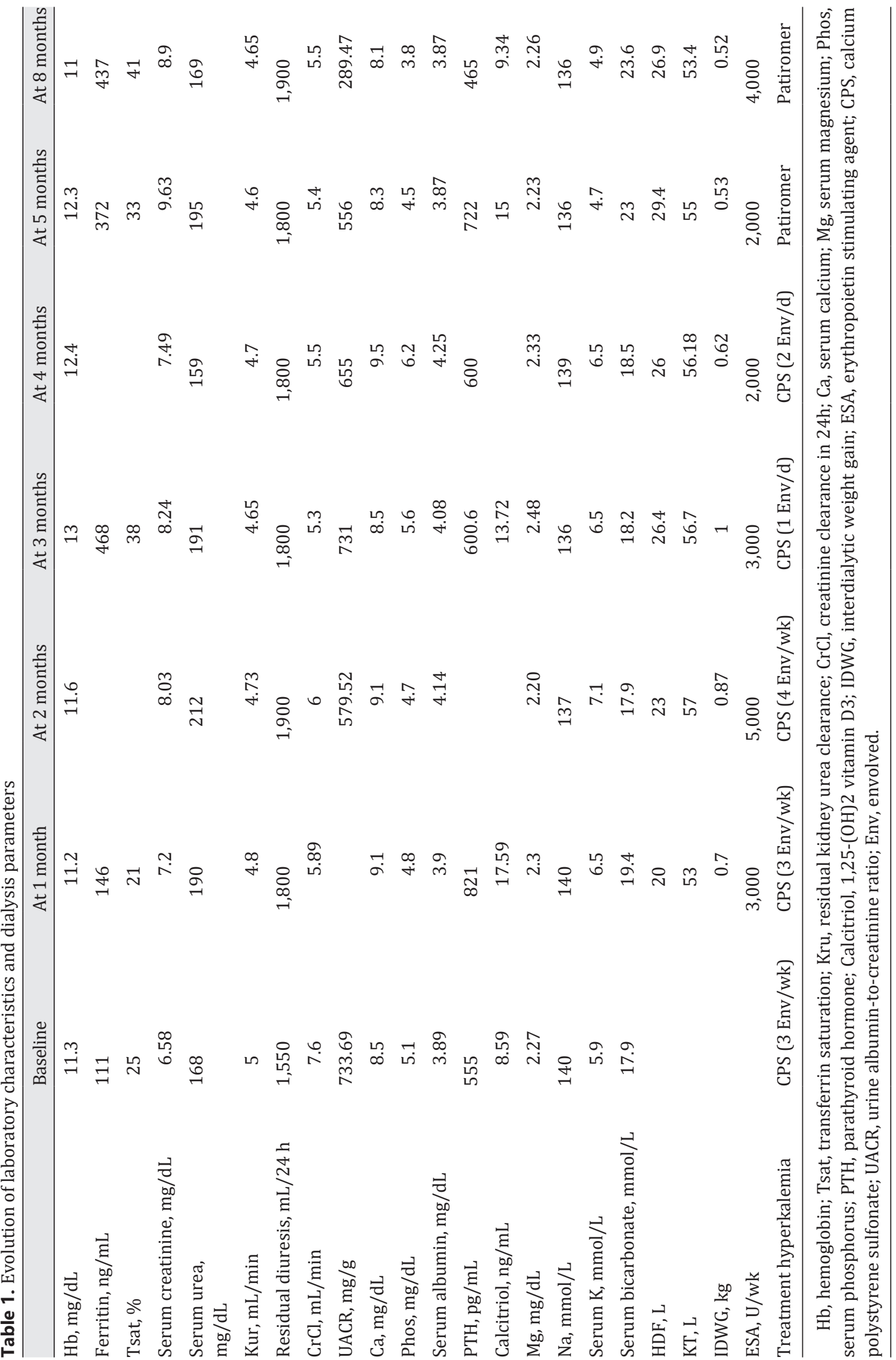


De La Flor et al.: Patiromer in a Patient with Severe Hyperkalemia on Incremental Hemodialysis

levels maintained below $5.0 \mathrm{mmol} / \mathrm{L}$. The analytic course is described in Table 1 . With the previously described measures, a progression to an iHD regimen of twice-a-week session could be avoided.

\section{Discussion}

The majority of HD patients lose their RKF. The physiological compensation mechanisms are limited, one of them being intestinal secretion [19]. The most frequent causes of hyperkalemia in HD patients include: excessive potassium ingestion, decreased intestinal secretion due to constipation or drugs that alter the intestinal secretion, and inadequate dialytic potassium exchange in patients that skip their HD sessions or reduce their sessions [20, 21]. Furthermore, redistribution of potassium from the intracellular compartment to the extracellular compartment can contribute to hyperkalemia. These alterations can be due to postdialysis rebound, drugs, endogenous insulin deficiency caused by prolonged fasting, metabolic acidosis, vascular access complications, pseudohyperkalemia caused by the rotational blood pump, other components of the extracorporeal circuit, or rhabdomyolysis [20, 22, 23].

Patiromer sorbitex calcium is a nonabsorbable polymer, which is free of sodium. It binds potassium in the colon, predominantly in the distal portion, reducing therefore the free potassium concentration and increasing the fecal excretion. This mechanism is associated with an exchange of potassium with calcium sorbitol. The efficacy and safety of patiromer for the management of hyperkalemia in patients with CKD not in HD and the concomitant treatment with RAASi in patients with type 2 diabetes mellitus and heart failure have been shown in 3 randomized clinical trials: PEARL-HF, OPAL-HK, and AMETHYST-DN. Patiromer showed to be efficacious for the reduction of serum $\mathrm{K}^{+}$up to a 52-week follow-up [24-26]. Side effects of patiromer include mainly mild GI symptoms, with constipation being the most frequent adverse effect [26]. Serious side effects have not been described, but it is important to monitor magnesium levels during the follow-up. In case of low magnesium levels, supplementation should be considered [25-28].

In the incremental dialysis expert opinion study by Kalantar-Zadeh et al. [29], urine volume $>0.5 \mathrm{~L}$ per day and a Kur of at least $3 \mathrm{~mL} / \mathrm{min}$ were 2 of 10 criteria that were mainly proposed to identify candidates for twice-weekly HD. Furthermore, apart from these 2 criteria based on RKF, the patient should meet 5 or more (out of 9) other criteria (i.e., intradialytic weight gain $2.5 \mathrm{~kg}$; stable cardiovascular status; infrequent hospitalization; satisfactory health-related quality of life; small to normal body size; good nutritional status; and absence of hyperkalemia, hyperphosphatemia, or profound anemia). These criteria are very similar to the criteria that we use in our center in order to choose patients for a once-a-week schedule. However, in our center protocol, the Kur must be $>4 \mathrm{~mL} / \mathrm{min}$ measured in RKF, as a main criteria for the patient inclusion. This is based on the design proposed by Deira et al. [7] in the IHDIP trial.

However, the HD prescription should be changed from twice a week to 3 times a week in cases where RKF is $<0.5 \mathrm{~L}$ day and/or Kur is $<2 \mathrm{~mL} / \mathrm{min}$ according to Kalantar-Zadeh et al. [29]. In our center, the progression criteria from 1 to 2 sessions a week are based on the decrease in Kur $<4$ and $>2.5 \mathrm{~mL} / \mathrm{min} / 1.73 \mathrm{~m}^{2}$; weekly weight gain that entails ultrafiltration rates $>13 \mathrm{~mL} / \mathrm{kg} / \mathrm{h}$, maintained for 3 weeks; and any clinical event that requires unscheduled sessions ( $>1$ ) for resolution such as hyperkalemia. In Spain, Merino et al. [12], described their experience of the first 8 years of application of an iHD of 2 sessions a week and its impact on the maintenance of RKF. Out of 15 patients included with a follow-up of no $<12$ months in iHD, 6 patients required conversion at $3 \mathrm{HD} /$ week due to laboratory parameters (hyperkalemia and high levels of phosphorus persistently) and 2 due to episodes of heart failure. In North America, the University of California, Irvine, was the first to systematically experiment with

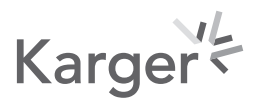


the practice of iHD twice a week. Ghahremani-Ghajar et al. [30] conducted a retrospective analysis of 13 incident ESRD patients who initiated incremental twice-weekly HD in the contemporary period of January 2015-August 2016. Among patients who eventually transitioned to thrice-weekly HD $(N=8)$, the 2 primary indications were problems with excessive intradialytic weight gain $(N=4)$ and inability to achieve adequate total clearance $(N=4)$. Among patients with inadequate total clearance, the following laboratory aberrancies were observed: hyperkalemia $(N=3)$, severe metabolic acidosis $(N=2)$, inadequate dialytic clearance per session $(N=4)$, and hyperphosphatemia $(N=2)$. That is why the management of hyperkalemia is crucial in our IHD program to avoid progression to more sessions per week.

Although patiromer is indicted in patients with ESRD in MHD, there exist only a few realworld postcommercialization studies that investigate the efficacy, safety, and tolerability of this drug. The majority of these studies are retrospective observational studies, and only a few research groups investigated its effect on patients undergoing MHD with thrice-weekly sessions, prospectively. No studies can be found in the literature that investigate the use of patiromer in iHD regimens, being our case one of the first to describe its efficacy and safety in patients with iHD of 1 session per week.

The studies of patiromer in patients undergoing MHD with thrice-weekly sessions allowed us to decide on the treatment with patiromer in our patient: Bushinsky et al. [16] studied 6 patients on MHD with moderate and severe hyperkalemia $\left(\mathrm{K}^{+} \geq 5.5\right.$ and $\left.\geq 6 \mathrm{mmol} / \mathrm{L}\right)$, with 5 of them being anuric. A 2-week follow-up was carried out, demonstrating that the use of patiromer (at a dose of $12.6 \mathrm{~g} /$ day) decreased the serum potassium levels and increased the fecal potassium excretion. In 2019, 1 retrospective observational cohort study with real-life data of patients in MHD was presented. Kovesdy et al. [17] used data of patients with hyperkalemia $\left(\mathrm{K}^{+} \geq 5.5 \mathrm{mmol} / \mathrm{L}\right)$ from the DaVita Clinical Research $(n=10,126)$ in order to analyze the change in serum potassium in patients with patiromer $(n=527)$, PSC $(n=852)$, and patients without chelates $(n=8,747)$. The majority of the patients (61\%) of the patiromer group initiated the drug in a dose of $8.4 \mathrm{~g} /$ day, and in $89 \%$ of the patients, this regimen was modified during follow-up. A statistically significant reduction in the serum potassium levels could be appreciated in this group (48 before vs. $22 \%$ after the initiation of patiromer had a $\mathrm{K}^{+} \geq 6.0 \mathrm{mmol} / \mathrm{L} ; p<0.001$ ). Furthermore, the authors concluded that patients in MHD and severe hyperkalemia showed a significant reduction in their potassium levels in long-term follow-up. Until the date of publication of this case report, our patient maintains treatment with patiromer at a dose of $8.4 \mathrm{~g}$ /day and has not presented any GI symptoms. Furthermore, the patient did not present any episodes of hyperkalemia.

The effect of patiromer on the electrolytic balance of potassium $\left(\mathrm{K}^{+}\right)$, sodium $\left(\mathrm{Na}^{+}\right)$, calcium $\left(\mathrm{Ca}^{+2}\right)$, magnesium $\left(\mathrm{Mg}^{+2}\right)$, and phosphate in patients undergoing MHD has recently been studied by Amdur et al. [18] for the first time. The authors of this study conducted a prospective crossed study in 3 phases ( 2 weeks of pretreatment, 12 weeks of patiromer with a dose of $16.8 \mathrm{~g} /$ day, and 6 weeks of posttreatment), in order to determine the efficacy, safety, and tolerability of patiromer for the reduction of serum potassium. Out of 219 anuric patients in MHD, 27 patients were selected for measuring of serum and fecal $\mathrm{K}^{+}, \mathrm{Na}^{+}, \mathrm{Ca}^{+2}, \mathrm{Mg}^{+2}$, and phosphate. The authors concluded that in patients undergoing MHD with hyperkalemia, patiromer at a dose of $16.8 \mathrm{~g}$ /day reduced serum potassium levels significantly at 12 weeks of treatment. This was associated with an increase in fecal potassium excretion, as a result of the binding of $\mathrm{K}^{+}$by the polymer in the GI tract. Every increase of $1 \mu \mathrm{g} / \mathrm{g}$ of fecal $\mathrm{K}^{+}$was associated with a decrease of $0.005 \mathrm{mmol} / \mathrm{L}$ of the serum potassium. Furthermore, the authors concluded that the serum calcium and fecal calcium increased during the treatment phase, which suggests that $\mathrm{Ca}^{+2}$ which is liberated by patiromer is later absorbed systematically. The reduction in serum magnesium was not associated with a change in fecal $\mathrm{Mg}^{+2}$ excretion, but did show a correlation with the serum potassium. No patient presented clinically significant hypokalemia, hypercalcemia, or hypomagnesemia during the follow-up. Our patient did not

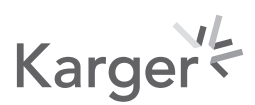


Case Reports

in Nephrology

and Dialysis

\begin{tabular}{l|l}
\hline Case Rep Nephrol Dial 2021;11:158-166 \\
\hline DOI: 10.1159/000516595 & $\begin{array}{l}\text { @ 2021 The Author(s). Published by S. Karger AG, Basel } \\
\text { www.karger.com/cnd }\end{array}$ \\
\hline
\end{tabular}

De La Flor et al.: Patiromer in a Patient with Severe Hyperkalemia on Incremental Hemodialysis

present any episodes of hypokalemia, and the $\mathrm{Na}^{+} \mathrm{Ca}^{+2}, \mathrm{Mg}^{+2}$, and $\mathrm{P}^{+2}$ levels remained normal. However, we were not able to determine changes in the fecal excretion of potassium, since we do not have availability for this kind of test in our center.

\section{Conclusion}

Cation-exchange resins, such as PSC, are not an effective and well-tolerated treatment for the chronic use in patients that undergo MHD. Furthermore, these drugs have important side effects on long-term use. However, patiromer has proven to be efficacious, safe, and well tolerated in HD patients with thrice-weekly sessions. Our case suggests that the use of patiromer may decrease the risks of hyperkalemia and improve tolerability of RAASi in patients with CKD treated with iHD with a once-a-week schedule.

\section{Statement of Ethics}

The research was conducted ethically in accordance with the World Medical Association Declaration of Helsinki. The patient signed an informed consent to collect anonymous data and for publishing her case (including publication of images).

\section{Conflict of Interest Statement}

The authors have no conflicts of interest to declare.

\section{Funding Sources}

The authors declare no financial support for the project.

\section{Author Contributions}

José Carlos de la Flor Merino, MD (primary author), conceived the ideas of the study; performed data collection, data analysis, and interpretation; provided revisions to scientific content of the manuscript. Javier Deira, $\mathrm{PhD}$, provided revisions to scientific content of the manuscript and access to crucial research components. Alexander Marschall, MD, performed data analysis, interpretation, and data collection. Francisco Valga, MD, provided revisions to scientific content of the manuscript and performed data collection. Tania Linares, MD, performed data analysis and interpretation. Tania Monzon, MD, provided revisions to scientific content of the manuscript. Cristina Albarracin, $\mathrm{PhD}$, provided revisions to scientific content of the manuscript and grammatical revisions to the manuscript. Elisa Ruiz, MD, provided revisions to scientific content of the manuscript and grammatical revisions to the manuscript.

\section{Availability of Data and Material}

The data used to support the findings of this study are available from the corresponding author on request (contact J.D.F., josedelaflor81@yahoo.com). 


\section{References}

1 Einhorn LM, Zhan M, Hsu VD, Walker LD, Moen MF, Seliger SL, et al. The frequency of hyperkalemia and its significance in chronic kidney disease. Arch Intern Med. 2009 Jun 22;169(12):1156-62.

2 Kovesdy CP, Regidor DL, Mehrotra R, Jing J, McAllister CJ, Greenland S, et al. Serum and dialysate potassium concentrations and survival in hemodialysis patients. Clin J Am Soc Nephrol. 2007 Sep;2(5):999-1007.

3 Iseki K, Uehara H, Nishime K, Tokuyama K, Yoshihara K, Kinjo K, et al. Impact of the initial levels of laboratory variables on survival in chronic dialysis patients. Am J Kidney Dis. 1996;28(4):541-8.

4 Rhee CM, Unruh M, Chen J, Kovesdy CP, Zager P, Kalantar-Zadeh K. Infrequent dialysis: a new paradigm for hemodialysis initiation. Semin Dial. 2013 Dec;26(6):720-7.

5 Mathew AT, Fishbane S, Obi Y, Kalantar-Zadeh K. Preservation of residual kidney function in hemodialysis patients: reviving an old concept. Kidney Int. 2016;90(2):262-71.

6 Rocco M, Daugirdas JT, Depner TA, Inrig J, Mehrotra R, Rocco MV, et al. KDOQI clinical practice guideline for hemodialysis adequacy: 2015 update. Am J Kidney Dis. 2015 Nov 1;66(5):884-930.

7 Deira J, Suárez MA, López F, García-Cabrera E, Gascón A, Torregrosa E, et al. IHDIP: a controlled randomized trial to assess the security and effectiveness of the incremental hemodialysis in incident patients. BMC Nephrol. 2019; 20(1):8.

8 Kaja Kamal RM, Farrington K, Wellsted D, Sridharan S, Alchi B, Burton J, et al. Impact of incremental versus conventional initiation of haemodialysis on residual kidney function: study protocol for a multicentre feasibility randomised controlled trial. BMJ Open. 2020 Aug 13;10(8):e035919.

9 Obi Y, Streja E, Rhee CM, Ravel V, Amin AN, Cupisti A, et al. Incremental hemodialysis, residual kidney function, and mortality risk in incident dialysis patients: a Cohort Study. Am J Kidney Dis. 2016 Aug 1;68(2):256-65.

10 Zhang M, Wang M, Li H, Yu P, Yuan L, Hao C, et al. Association of initial twice-weekly hemodialysis treatment with preservation of residual kidney function in ESRD patients. Am J Nephrol. 2014 May 22;40(2):140-50.

11 Fernández-Lucas M, Teruel-Briones JL, Gomis-Couto A, Villacorta-Pérez J, Quereda-Rodríguez-Navarro C. Maintaining residual renal function in patients on haemodialysis: 5-year experience using a progressively increasing dialysis regimen. Nefrologia. 2012;32(6):767-76.

12 Merino JL, Domínguez P, Bueno B, Amézquita Y, Espejo B, Paraíso V. Aplicación de una pauta de hemodiálisis incremental, basada en la función renal residual, al inicio del tratamiento renal sustitutivo. Nefrología. 2017; 37(1):39-46.

13 Nasir K, Ahmad A. Treatment of hyperkalemia in patients with chronic kidney disease: a comparison of calcium polystyrene sulphonate and sodium polystyrene sulphonate. J Ayub Med Coll Abbottabad. 2014; 26(4):455-8.

14 Lepage L, Dufour AC, Doiron J, Handfield K, Desforges K, Bell R, et al. Randomized clinical trial of sodium polystyrene sulfonate for the treatment of mild hyperkalemia in CKD. Clin J Am Soc Nephrol. 2015 Dec 7;10(12): 2136-42.

15 Palmer BF. Potassium binders for hyperkalemia in chronic kidney disease-diet, Renin-Angiotensin-Aldosterone system inhibitor therapy, and hemodialysis. Mayo Clin Proc. 2020;95(2):339-54.

16 Bushinsky DA, Rossignol P, Spiegel DM, Benton WW, Yuan J, Block GA, et al. Patiromer decreases serum potassium and phosphate levels in patients on hemodialysis. Am J Nephrol. 2016 Nov 1;44(5):404-10.

17 Kovesdy CP, Rowan CG, Conrad A, Spiegel DM, Fogli J, Oestreicher N, et al. Real-world evaluation of patiromer for the treatment of hyperkalemia in hemodialysis patients. Kidney Int Rep. 2019 Feb 1;4(2):301-9.

18 Amdur RL, Paul R, Barrows ED, Kincaid D, Muralidharan J, Nobakht E, et al. The potassium regulator patiromer affects serum and stool electrolytes in patients receiving hemodialysis. Kidney Int. 2020 Nov 1;98(5):133140 .

19 Sandle GI, Gaiger E, Tapster S, Goodship THJ. Evidence for large intestinal control of potassium hemoeostasis in uraemic patients undergoing long-term dialysis. Clin Sci. 1987;73(3):247-52.

20 Kim HJ. Pathogenesis and treatment of dyskalemia in maintenance hemodialysis and CAPD. Electrolyte Blood Press. 2006;4:47-52.

21 Khalil AA, Frazier SK, Lennie TA, Sawaya BP. Depressive symptoms and dietary adherence in patients with end-stage renal disease. J Ren Care. 2011 Mar;37(1):30-9.

22 Ngugi NN, McLigeyo SO, Kayima JK. Treatment of hyperkalaemia by altering the transcellular gradient in patients with renal failure: effect of various therapeutic approaches. East Afr Med J. 1997 Aug;74(8):503-9.

23 Edwards NC, Steeds RP, Stewart PM, Ferro CJ, Townend JN. Effect of spironolactone on left ventricular mass and aortic stiffness in early-stage chronic kidney disease. A randomized controlled trial. J Am Coll Cardiol. 2009 Aug 4;54(6):505-12.

24 Buysse JM, Huang IZ, Pitt B. PEARL-HF: prevention of hyperkalemia in patients with heart failure using a novel polymeric potassium binder, RLY5016. Future Cardiol. 2012;8:17-28.

25 Weir MR, Bakris GL, Gross C, Mayo MR, Garza D, Stasiv Y, et al. Treatment with patiromer decreases aldosterone in patients with chronic kidney disease and hyperkalemia on renin-angiotensin system inhibitors. Kidney Int. 2016 Sep 1;90(3):696-704.

26 Pitt B, Garza D. The tolerability and safety profile of patiromer: a novel polymer-based potassium binder for the treatment of hyperkalemia. Expert Opin Drug Saf. 2018 May 4;17(5):525-35.

27 Bakris GL, Pitt B, Weir MR, Freeman MW, Mayo MR, Garza D, et al. Effect of patiromer on serum potassium level in patients with hyperkalemia and diabetic kidney disease. JAMA. 2015 Jul 14;314(2):151-61. 
Case Reports

in Nephrology

and Dialysis
Case Rep Nephrol Dial 2021;11:158-166 (c) 2021 The Author(s). Published by S. Karger AG, Basel www.karger.com/cnd

De La Flor et al.: Patiromer in a Patient with Severe Hyperkalemia on Incremental Hemodialysis

28 Weir MR, Bakris GL, Bushinsky DA, Mayo MR, Garza D, Stasiv Y, et al. Patiromer in patients with kidney disease and hyperkalemia receiving RAAS inhibitors. N Engl J Med. 2015 Jan 15;372(3):211-21.

29 Kalantar-Zadeh K, Unruh M, Zager PG, Kovesdy CP, Bargman JM, Chen J, et al. Twice-weekly and incremental hemodialysis treatment for initiation of kidney replacement therapy. Am J Kidney Dis. 2014;64(2):181-6.

30 Ghahremani-Ghajar M, Rojas-Bautista V, Lau WL, Pahl M, Hernandez M, Jin A, et al. Incremental hemodialysis: the University of California Irvine experience. Semin Dial. 2017;30(3):262-9. 